\title{
Eficiencia del transporte de carga internacional mexicano: análisis por entidad federativa a través del Índice Malmquist, 2010-2014*
}

\author{
The Efficiency of Mexican International Freight Transport: Analysis by Federative \\ Entity Through Malmquist Index, 2010-2014
}

América Ivonne Zamora Torres ${ }^{* *}$ y Juan González García ${ }^{* * *}$

\begin{abstract}
RESUMEN
Un eslabón crucial en el comercio exterior de México, es el transporte de carga internacional carretero, el cual se presupone eficiente per se, lo que no siempre sucede en la realidad. De ahí la importancia de estudios que midan el grado de eficiencia del mismo. La presente investigación tiene como objetivo identificar el grado de eficiencia o ineficiencia del sistema de transporte carretero de carga internacional de México, así como determinar qué factores incidieron en el cambio tecnológico en cada uno de los estados de la República mexicana en el periodo de 2010-2014, para lo cual se utilizó la metodología de la Envolvente de Datos (DEA) y el Î́ndice Malmquist. De acuerdo con los resultados de la medición de la eficiencia realizada en esta investigación, las entidades federativas con el sistema carretero de carga que experimentaron un mayor cambio tecnológico fueron: Chihuahua, Sinaloa, Guanajuato, Estado de México y Querétaro. Los resultados permiten afirmar que los procesos de innovación y cambio tecnológico en el sistema carretero de transporte de carga, constituyen una condición sine quanon para la mejora del comercio exterior mexicano.
\end{abstract}

Palabras clave: Eficiencia, transporte, exportaciones, logística, infraestructura.

Clasificación JEL: C43, C67.

\begin{abstract}
A crucial link in the international trade of Mexico is the international road freight transport, which is presupposed efficient per se, which doesn't always happen in reality. Hence the importance of studies that measure the degree of efficiency of it. This research aims to identify the degree of efficiency or inefficiency of the system of international road freight transport of Mexico, as well as to determine which factors contribute and determine technological change in each of the States of the Mexican Republic for the period 2010-2014, in order to achieve that, it uses Data Envelopment Analysis (DEA) and Malmquist Index. According to the results of the efficiency measurement implemented in this research, the entities with the road freight system that experienced the greatest technological change were: Chihuahua, Sinaloa, Guanajuato, State of Mexico and Queretaro. The results allow to affirm that the processes of innovation and technological change in the road freight transport system constitute a sine quanon condition towards improving Mexico's foreign trade.
\end{abstract}

Keywords: Efficiency, transport, export, logistics, efficiency, infrastructure. JEL Classification: C43, C67.

\footnotetext{
* Fecha de recepción: 26/07/2017. Fecha de aceptación: 03/05/2018.

** Universidad Michoacana de San Nicolás de Hidalgo, México. E-mail: azamora@umicm.mx. ORCID: 0000-00031811-4711.

*** Universidad de Colima, México. E-mail: jgogar@ucol.mx. ORCID: 0000-0003-1458-8047.
} 


\section{INTRODUCCIÓN}

Si bien la teoría pura del comercio internacional clásica y neoclásica se especializó en la dotación de factores productivos y los determinantes principales que inciden en el comercio internacional de los países, es obvio que existe un sinnúmero de factores no productivos, en el sentido literal de la palabra, que inciden directamente en el objetivo de incentivar el comercio internacional de los países. En este sentido, existen diferentes estudios que analizan los determinantes del transporte internacional y su importancia para realizar, a costos competitivos, las operaciones comerciales, que tienen que ver con el comercio internacional.

En estudios recientes (Gonzalez, Guasch, y Serebrisky (2007); Sarmento, Renneboog y Verga (2017)), se destaca la importancia de los costos logísticos, incluidos los costos de transporte que, por ejemplo, para el caso de América Latina, representan entre el 18 por ciento y el 32 por ciento del valor monetario total del producto; porcentaje muy alto, en comparación con los países miembros de la Organización para la Cooperación y el Desarrollo Económico (OCDE). En efecto, el costo logístico del conjunto de países de la OCDE, se encuentra en un promedio del 9 por ciento, menor incluso al de Estados Unidos de América (EUA) que es del 9.5 por ciento. Martínez (2008), por su parte, enfatiza la importancia de la variable del gasto logístico concluyendo que en países en desarrollo puede superar el 20 por ciento del valor del producto final.

Cipoletta, Pérez, y Sánchez (2010), realzan la influencia que tienen las variables medioambientales en el transporte y la calidad de la infraestructura del transporte. Ésta se considera una variable primordial en el estudio de la eficiencia del transporte. Wilmsmeier y Sanchez (2009) y Arvis J. (2007) concluyen que la infraestructura del transporte juega un rol central en términos de eficiencia. Mientras que, para Choi, Roberts y Lee (2015) el transporte es una parte importante del desarrollo y crecimiento de las actividades económicas.

Asimismo, una variable implícita, de reciente relevancia, sobre todo para México, está relacionada con la seguridad en el transporte. En efecto, la Asociación Nacional de Transporte Privado (ANTP, 2018) afirma que: "las empresas en México destinan del 15 por ciento al 20 por ciento a gastos inherentes a la seguridad, aunque en la mayoría de los casos, dicho monto no se manifiesta en una mejora en la seguridad". También, desde inicios de la presente década, los principales factores de riesgo logístico en México son: el narcotráfico (con un 30 por ciento) y el robo (con un 25 por ciento) (Cervantes, 2011).

Desde otra perspectiva, pero intrínsecamente vinculada a la operatividad del transporte, destaca por su importancia la variable tecnológica, que cotidianamente impacta en el desempeño de diversos sectores de la economía. En el actual siglo (XXI) la tecnología se está convirtiendo en una variable clave, 
que genera diferencias en el desempeño y/o rendimiento de los agentes económicos y de las regiones y territorios. Esto se constata en el caso del sector carretero mexicano en donde, en la presente década y de manera particular, en el periodo 2010-2014, las entidades que mayor eficiencia técnica presentan son las entidades que más han invertido en incorporar los desarrollos e innovaciones tecnológicas en su operatividad.

Por otra parte, la constante competencia internacional por los mercados exige el cumplimiento de plazos y tiempos exactos en la entrega de bienes y servicios a nivel local e internacional. Dussel (2008), realiza un análisis de los procesos de exportación de varias empresas mexicanas, donde la principal limitación del transporte del comercio exterior mexicano es la incertidumbre en términos de tiempo, ya que se requieren diversos permisos, revisiones exhaustivas de la mercancía en aduanas, así como otros procesos de verificación de documentos y la propia mercancía, produciendo un alto nivel de incertidumbre en el tiempo de entrega del producto a los importadores. Djankov et al. (2007), afirman que cada día adicional que el producto requiere para ser transportado implica una caída de 1 por ciento en el comercio, con las consecuentes afectaciones que esto tiene sobre la producción y consumo.

Desde otra perspectiva, pero de relevancia también reciente, no sólo nacional sino internacional, se encuentran los costos relacionados con el medioambiente. Zhang, Zhou y Kung (2015), utilizando el Índice Malmquist, midieron la incidencia de las emisiones de carbono dentro del sistema de transporte chino, constituyéndose su estudio en un referente para la medición de la eficiencia relacionada con las variables ambientales. Kim \& Van Wee (2009), realizaron una evaluación de las emisiones de bióxido de carbono $\left(\mathrm{CO}_{2}\right)$ para los sistemas intermodales de mercancías mediante la utilización del ferrocarril y del transporte terrestre en Europa, concluyeron que el sistema ferroviario ha sido el más amigable con el medio ambiente, proponiendo la utilización de los sistemas intermodales de mercancías basados en el ferrocarril, debido a que emiten menos $\mathrm{CO}_{2}$. Asimismo, Beltrán y Picazo-Tadeo (2015) analizan el cambio ambiental en la industria del transporte de 38 países por medio del Índice Malmquist obteniendo, entre sus resultados, un mejoramiento del desempeño ambiental desde 1990, lo que es explicado principalmente a través de las eco-innovaciones.

Finalmente, la Agencia Internacional de Energía (IEA/OECD, 2009), indica que el 19 por ciento del consumo mundial de energía es resultado de la utilización del transporte, generando el 23 por ciento de las emisiones de $\mathrm{CO}_{2}$ relacionadas con la energía. Su pronóstico, en caso de continuar con la misma tendencia de crecimiento sería del 50 por ciento para el año 2030 y del 80 por ciento para el año 2050. 
La estructura del artículo será la siguiente: además de esta breve introducción general, se presentarán las siguientes secciones: una de evidencia empírica y metodología, para justificar la propuesta de análisis para medir la eficiencia técnica del subsector de autotransporte de carga; posteriormente, pondrá una definición básica de eficiencia, diferenciando lo que es la eficiencia técnica de la económica. Una tercera sección, explicará el Modelo y/o Índice Malmquist, previo a la exposición y antecedentes de lo que es el Índice Malmquist; otra sección explica propiamente el Índice Malmquist que será empleado para hacer el cálculo de la eficiencia de las entidades de la república en infraestructura y tecnología del subsector autotransporte de carga; en las secciones finales, se describe el Modelo para obtener el Índice Malmquist de las entidades de la república; se discuten los resultados y finalmente se formulan algunas reflexiones que engloban lo desarrollado y permiten soportar, teórica y técnicamente, nuestras propuestas de mejora en la eficiencia portuaria nacional.

\section{EVIDENCIA EMPÍRICA Y METODOLOGÍA}

Desde nuestro punto de vista, para insertarse exitosamente en los flujos globales de comercio internacional, es determinante el tipo de operación elegida por el sector transporte de cada país. En efecto, en los países desarrollados existe una mayor diversificación debido a las fuentes de financiamiento, lo que le ha permitido una rápida adaptación al cambio en cuestión de licencias, equipos de seguridad, renovación de unidades, entre otros. Ello, contrasta con la regulación mexicana del transporte de carga, que más que vigorizar al subsector, se ha convertido en el principal obstáculo para la eliminación de las barreras al comercio (Mendoza y Díaz, 2003).

Por otra parte, si se considera la evolución reciente de las unidades de transporte de carga en 2011, México contaba con 689,000 unidades de transporte registradas (Estadísticas de Transporte de América del Norte, 2011). Sin embargo, a pesar de este gran número de unidades de transporte terrestre, la estructura de mercado en el sector transporte en México es de carácter oligopólico, donde las grandes empresas operadoras del servicio de transporte desarrollan estrategias designadas a restringir la operación de su competencia (Medina, 2011).

El sector del autotransporte de carga mexicano se encuentra dividido en dos segmentos, el primero se refiere a las empresas del sector transporte de capacidad pequeña, que se encarga de cubrir distancias cortas, bajo el esquema de hombre camión (operadores que manejan sus propias unidades). Por otra parte, se encuentran las grandes empresas propietarias de un número mayor de unidades, mismas que ofrecen un servicio especializado mediante el uso de 
contenedores refrigerados, unidades especiales para traslado de materiales y residuos peligrosos, entre otros. Estas características o condiciones, confirman la estructura oligopólica del mercado de autotransporte de carga el cual, a priori, está impidiendo el surgimiento de la eficiencia global del sector.

El gobierno federal, para tratar de atender parte de la problemática global del subsector del autotransporte, estableció en el Programa Sectorial de Comunicaciones y Transportes 2013-2018, un diagnóstico general para enfrentar muchos de los retos que no han sido cubiertos en el pasado debido a una falta de visión integral en el diseño e implementación de acciones y proyectos (SCT, 2013). Dentro de los retos que se señalan en dicho documento sobresalen la seguridad, el desarrollo regional, la conectividad, el medio ambiente y el desarrollo urbano. De hecho, gran parte del proyecto de inversión pública del gobierno federal del presente sexenio, tuvo como baluarte la inversión en infraestructura.

De acuerdo con los informes del Sector Comunicaciones y Transportes (2016) las cinco entidades federativas que, en el año 2015, contaban con una mayor densidad de red carretera, fueron: Tlaxcala, Estado de México, Morelos, Colima e Hidalgo; mientras que las entidades de menor densidad, fueron: Chihuahua, Ciudad de México, Coahuila de Zaragoza, Baja California Sur y Campeche.

\section{II.1. Nociones de eficiencia}

La presente investigación tiene como finalidad la medición de la obtención de un Índice de eficiencia en el sector del transporte terrestre. Para fines del presente estudio, nos referiremos a la eficiencia retomando el concepto propuesto por Bardhan (1995), quien la define como: "la alusión a la capacidad de obtener objetivos, por medio de una relación deseable entre inputs y outputs".

Ahora bien, es importante destacar que inicialmente la noción previa de eficiencia fue propuesta por Farell (1957), para quien cada unidad de producción es evaluada en relación con otras tomadas a partir de un grupo, que para nuestro caso son las entidades federativas. De tal forma que las medidas de eficiencia son relativas y no absolutas, siendo el valor alcanzado una expresión de la desviación observada respecto de la curva donde se encuentran las unidades consideradas eficientes dado los inputs y outputs.

En el sentido clásico, la eficiencia, medida como el grado mínimo de recursos utilizados asociado a un máximo nivel de outputs generados, logra un óptimo de Pareto, bajo ciertas condiciones dadas como pueden ser el nivel de precios y la tecnología. De este tipo de análisis se desprende una frontera o limite, derivado de las observaciones realizadas de cada una de las unidades consideradas que pueden ser empresas, instituciones, países, regiones, etcétera. 
Conviene aclarar que cuando dicho óptimo está definido por la función de producción, a la medida de eficiencia que se obtiene se le denomina eficiencia técnica. Mientras que, si la comparación se realiza considerando un óptimo de índole económica (como pueden ser costos, maximización de ingresos o beneficios), a la medida de eficiencia que resulta de aplicar el modelo se le denomina eficiencia económica.

Dado que la presente investigación se realizó considerando como unidades de comparación las diferentes administraciones aduaneras, y dado que el fin último de éstas no es perseguir una maximización de ingresos sino más bien el adecuado uso de los recursos gubernamentales para mejorar la productividad de las actividades realizadas en las aduanas, y por ende generar una mayor facilitación del comercio internacional, se realizará la estimación del cambio productivo utilizando el Índice de Malmquist (1953).

\section{MODELO E ÍNDICE DE MALMQUIST}

Fue Moortenn en 1961, quien empleó la técnica, metodología o Índice de Malmquist, para la medición y determinación de la eficiencia; buscaba comprobar cómo un input puede ser deflactado para producir el nivel observado de output del otro periodo.

Caves, Christensen y Diewert en 1982 establecieron la relación entre los índices de Malmquist (1953) y Törnqvist (1936) desarrollando el Índice de Malmquist a través de dos enfoques. El primero, analizando las diferencias de productividad como las diferencias en el máximo output alcanzable dado cierto nivel de inputs conocido como Índice de Malmquist de productividad basado en el output; mientras que el segundo enfoque analiza el nivel de productividad, como consecuencia de las diferencias en el mínimo nivel de inputs que permite producir ciertos niveles de outputs determinados, siendo este el Índice de Malmquist de productividad basado en el input.

Berg, Forsund y Jansen (1992) hicieron la conexión entre los conceptos de función de distancia y las medidas de eficiencia de Farrell (1957). A partir de ahí se pudieron observar las unidades analizadas ineficientes, sustituyendo el concepto de frontera tecnológica por el de frontera de tecnología.

El modelo que se utilizará en el presente trabajo es el Modelo o Índice de Malmquist de productividad basado en el input. La justificación que nos permite optar por este índice, se debe a que, al ser las unidades de análisis organismos públicos, se parte de que el máximo beneficio tendría que ser un mayor flujo comercial o, dicho de otro modo, una mayor entrada y salida de productos en los diferentes territorios nacionales, por lo que no cabría esperar una reducción 
o un output estable sino, por el contrario, una minimización de los recursos empleados por el gobierno en la administración aduanera, lo que se traduciría teóricamente en un aumento de recursos disponibles en otros sectores que también coadyuven al crecimiento económico.

Adicionalmente acorde con Grifell y Lovell (1993a), a pesar de que la exogeneidad no es un problema estadístico en DEA, en el sentido que lo es para los modelos econométricos, la elección entre las medias orientadas en inputs o en outputs se someten a las mismas consideraciones. Por lo que la administración aduanera de los diferentes países objeto de estudio están sometidas a condiciones de demanda, ajustando libremente sus inputs, el modelo orientado a los inputs sería así, el más apropiado.

Cabe señalar que existen numerosos estudios que evalúan la productividad y/o eficiencia del transporte utilizando el Índice Malmquist, dentro los que destacan el trabajo realizado por Choi, Roberts y Lee (2015) que analizan la productividad del sistema de transporte aéreo, férreo, marítimo y carretero de las cinco compañías más grandes en Estados Unidos para el periodo de 2004 al 2011. De dicho estudio se desprende el hecho de que la industria del transporte en Estados Unidos ha tenido un fuerte crecimiento positivo a excepción de los años 2008, 2009 y 2010. Çipil (2016), a través del Índice de Malmquist, realiza un análisis de eficiencia de 12 regiones del sistema de transporte aéreo. Schoyen y Odeck (2015) realizan un estudio utilizando esta misma técnica para medir la eficiencia de seis terminales de contenedores en países nórdicos y Reino Unido, mostrando una mayor eficiencia del sistema de transporte portuario de los países nórdicos. Finalmente, Wanke, Chen, Moreira y Barros (2018) analizan la productividad Malmquist de los 17 puertos chinos más importantes.

\section{II.1. Indice Malmquist}

Para ilustrar el cálculo del Índice de Malmquist, supóngase que la función de trasformación que describe la tecnología de la firma en cada periodo es:

$$
F_{t}\left(Y^{t}, X^{t}\right)=0 \quad \mathrm{t}=1, \ldots, T
$$

Donde $y^{t}=\left(y_{1_{1}}^{t}, \ldots, y_{N}^{t}\right) \in R_{N}^{+}$es el vector de outputs y $x^{t}=\left(x_{1}^{t}, \ldots, x_{M}^{t}\right) \in R_{M}^{+}$denota el vector de inputs correspondientes ambos al periodo $t$.

La tecnología puede ser representada de manera formal a través de la "función distancia de input" utilizada por Caves et al. (1982): 


$$
D^{r}\left(y^{s}, x^{s}\right)=\operatorname{Max} \mu_{r s}\left[\mu_{r s}: F_{r}\left(y^{s}, \frac{x^{s}}{\mu_{r s}}\right)=0\right] r, s=1, \ldots, T ; r<s
$$

En donde el escalar $\mu_{r s}$ es la máxima deflación del vector de inputs del periodo $s\left(x^{s}\right)$ tal que el vector inputs deflactado resultante $x^{s} / \mu_{r s}$ y el vector de outputs estén en la frontera del período $r$. Si $r=s$ se está comprando cada empresa con la frontera del periodo al que pertenece, por lo que la función distancia de input $D^{r}\left(y^{r}, x^{r}\right) \geq 1$ siendo igual a la unidad en el caso de que la empresa evaluada sea eficiente y, por tanto, se encuentre en la frontera. Por el contrario, si $r \neq s$ la función distancia puede tomar valores inferiores a la unidad, ya que la observación pertenece a un periodo diferente del de la frontera con la cual se está comparando (frontera de referencia).

El Índice de Malmquist de productividad basado en los inputs, tomando la tecnología del periodo $r$ como referencia, se define como:

$$
M^{r}\left(y^{s}, x^{s}, y^{s}, x^{r}\right)=\frac{D^{r}\left(y^{r}, x^{r}\right)}{D^{r}\left(y^{s}, x^{s}\right)}
$$

Un $M_{r}>1$ indica que la productividad del periodo s es superior a la del periodo $r$, puesto que la deflación necesaria del vector de inputs del periodo $r$ para estar en la frontera del periodo $r$ es superior a la aplicable al vector de inputs del período $s$ para que esté en la frontera del periodo $r$. Por el contrario, un $M_{r}$ índica que la productividad ha descendido entre los periodos $r$ y $s$.

Cabe aclarar que los números índices han sido frecuentemente utilizados para analizar el cambio productivo. Los más utilizados son el Índice de Fisher (1922); el de Törnqvist (1936), y el de Malmquist (1953). Las ventajas de la utilización de los números índices del tipo Fisher y Törnqvist es que pueden ser calculados sin recurrir a la estimación de la tecnología subyacente, ya que únicamente precisan datos de cantidades (de outputs o de inputs) y de precios.

Como señalan Grifell et al. (1993a), el Índice de Malmquist presenta tres ventajas frente al de Fisher y Törnqvist. En primer lugar, no necesita suponer comportamiento minimizador de costes o maximizador de ingresos. En segundo lugar, no precisa de datos relativos o precios, lo cual es una gran ventaja, sobre todo en aquellos casos en los que existan graves carencias estadísticas, $o$ simplemente en los casos en los que la existencia de regulaciones sobre los mismos y/o presencia de poder de mercado reflejado en los precios hagan inviable su utilización. Por último, permite la descomposición del cambio productivo en cambio en la eficiencia técnica (catching-up) y cambio técnico (o desplazamiento de la frontera), siendo éste el objetivo central del presente trabajo. Quizá un inconveniente que presenta el Índice Malmquist es que, para su cálculo individual, 
precisa, según se ha visto, el previo cálculo de la distancia, por lo que requiere la estimación de la función de producción.

La descomposición del cambio productivo, en cambio en la eficiencia técnica y progreso (regreso) técnico, fue una cuestión abordada por Nishimizu y Page (1982). Estos autores analizaron el sector industrial de la antigua Yugoslavia en el periodo 1965-1978 mediante la especificación y posterior estimación por métodos de programación matemática, de una función de producción translong imponiendo rendimientos constantes a escala.

Después de este trabajo pionero, Berg et al. (1992) obtienen una similar descomposición del cambio productivo utilizando el Índice de Malmquist. Para su estimación emplean la técnica no paramétrica determinista DEA, mucho más flexible que la técnica paramétrica empleada por Nishimizu et al. (1982).

Desde entonces, las aportaciones empíricas y teóricas más relevantes corresponden a los trabajos de Grifell et al. (1993a) que aplican la metodología de Berg et al. (1992) para analizar el cambio productivo de las cajas de ahorro españolas. Grifell et al. (1993b) proponen una descomposición alternativa que permite analizar adicionalmente la posible presencia de sesgo tecnológico, y Grifell et al. (1996) demuestran que el Índice de Malmquist ofrece una medida imprecisa del cambio productivo cuando los rendimientos a escala no son constantes.

La referida descomposición del Índice de Malmquist en el efecto catching-up y desplazamiento de la frontera puede expresarse como (Berg et al. (1992) y Grifell et al. (1993a, b y 1996)):

$$
M_{r}\left(y^{s}, x^{s}, y^{r}, x^{r}\right)=\frac{D^{r}\left(y^{r}, x^{r}\right)}{D^{r}\left(y^{s}, x^{s}\right)}=\frac{D^{r}\left(y^{r}, x^{r}\right)}{D^{r}\left(y^{s}, x^{s}\right)} \cdot \frac{D^{s}\left(y^{s}, x^{s}\right)}{D^{r}\left(y^{s}, x^{s}\right)}
$$

El primer cociente representa el acercamiento de las empresas a la frontera ocurrido entre los periodos $r$ y $s$, mientras que el segundo término muestra el desplazamiento relativo de la frontera entre los dos periodos.

Si la empresa se encuentra en ambos periodos en sus fronteras respectivas, el primer término será igual a 1 y el cambio productivo experimentado entre los dos periodos vendrá explicado únicamente por el movimiento de la frontera. Por el contrario, si el segundo término es 1 (la frontera no se ha desplazado), los cambios de productividad estimados por $M_{r}$ vendrán explicados únicamente por los cambios en la eficiencia de las empresas en ambos periodos (catching-up). En los demás casos, los cambios productivos reflejados en $M_{r}$ serán una mezcla de cambios en la eficiencia y desplazamientos de la frontera. 
Färe y Lovell (1978) formalizaron la relación existente entre la función distancia de input y las medidas de Farell ahorradoras de inputs $E_{r_{r}}\left(y^{r}, x^{r}\right)$, y demostraron que la función distancia es igual a la inversa de la medida de Farell ahorradora de inputs

$$
D^{r}\left(y^{r}, x^{r}\right)=\left[E_{r_{r}}\left(y^{r}, x^{r}\right)\right]^{-1}
$$

Dado que, en el caso de rendimientos constantes a escala, se cumple que $\frac{x_{r}^{s}}{x_{r r}}=y^{s} / y^{r}$ el Índice de Malmquist puede ser escrito en este caso como:

$$
M_{r}\left(y^{s}, x^{s}, y^{r}, x^{r}\right)=\frac{D^{r}\left(y^{r}, x^{r}\right)}{D^{r}\left(y^{s}, x^{s}\right)}=\frac{E_{r s}}{E_{r r}}=\frac{\frac{x_{r}^{s}}{x_{s}^{r}}}{x_{r}^{r}}=\frac{\frac{y^{s}}{x^{s}}}{\frac{y^{r}}{x^{r}}}
$$

Que en este caso se reduce a un simple ratio de índices de productividad de los periodos $r$ y $s$.

Para este ejemplo, la descomposición del Índice de Malmquist genera el efecto catching-up (CU) y el cambio técnico o desplazamiento de la frontera (DF), que puede expresarse como:

$$
M_{r}\left(y^{s}, x^{s}, y^{r}, x^{r}\right)=\frac{E_{r s}}{E_{r r}}=\frac{E_{s s}}{E_{r r}} \cdot \frac{E_{r s}}{E_{s s}}=C U\left(y^{s}, x^{s}, y^{r}, x^{r}\right) \cdot D F\left(y^{s}, x^{s}, y^{r}, x^{r}\right)
$$

En donde el catching-up o acercamiento relativo a la frontera ocurrido entre el periodo $r$ y $s$ sería $\left.C U\left(y^{s}, x^{s}, y^{r}, x^{r}\right)=\frac{E_{s s}}{E_{r}}=\left(x_{s}^{s} / x_{s}\right) / x_{r}^{r} / x^{r}\right)^{8}$ y el desplazamiento de la frontera entre los dos periodos, vendria expresado por

$D F\left(y^{s}, x^{s}, y^{s}, x^{s}\right)=\frac{E_{r s}}{E_{s s}}=\left(x_{r}^{s} / x^{s}\right) /\left(x_{s}^{s} / x^{s}\right)=x_{r}^{s} / x_{s}^{s}$

\section{ESPECIFICACIONES DEL MODELO}

Antes de utilizar un modelo DEA como herramienta para medir la eficiencia, es necesario tomar en consideración diferentes aspectos tales como la orientación del modelo, si se considerarán rendimientos constantes o variables a escala y la selección de inputs y outputs a utilizar, así como el número de los mismos. Esto es necesario para poder utilizar un modelo con orientación output en el cálculo del Índice Malmquist, ya que se pretende maximizar las exportaciones con los insumos que se tienen por lo que resulta más adecuado utilizar un modelo con orientación output. 
Los modelos DEA pueden asumir rendimientos constantes de escala (CRS) o rendimientos variables de escala (VRS). Sin embargo, no es posible determinar con un buen nivel de confianza antes de seleccionar un modelo en lugar de otro, cuál es el más apto. Por esta razón algunos autores afirman que es recomendable realizar pruebas con ambos modelos (CRS y VRS). Cuando los resultados de ambos modelos coinciden en gran medida es entonces mejor utilizar el modelo de rendimientos constantes de escala (CRS), de lo contrario es más adecuado utilizar rendimientos variables de escala (VRS) (Mahammady, 2006). Una vez realizados los cálculos con ambos modelos, se seleccionó el modelo DEA de rendimientos constantes de escala (VRS) como el más adecuado para el presente estudio.

Otra de las limitantes de los modelos DEA es el número total de inputs y outputs que se pueden utilizar para el análisis. Específicamente, es recomendable que el número de DMUS a ser analizadas (en la presente investigación entidades federativas) sea al menos tres veces más grande que el número en suma de inputs y outputs incluidos en el análisis (Cooper, Seiford y Tone, 2006). Esta limitante implica, para empezar, un problema al tratar de seleccionar los inputs y outputs más relevantes, para lo cual se consultó información de los reportes de la Organización Mundial de Comercio (OMC) y el SCCP, principalmente, de donde se seleccionarán los siguientes inputs y outputs:

\section{III.1. Inputs}

Los inputs seleccionados se consideraron tomando en cuenta cuatro variables principales $a$ ) la infraestructura carretera; $b$ ) la infraestructura de las empresas transportistas; $c$ ) recursos humanos y $d$ ) calidad. De dichas variables se desprenden los inputs a utilizar considerando el cambio tecnológico en la infraestructura carretera, de las empresas transportistas y estándares de calidad, así como capacitación en los recursos humanos. Quedando de la siguiente manera:

a) Infraestructura carretera

1. Carreteras pavimentadas

2. Densidad vehicular de carga

b) Infraestructura de empresas transportistas

3. Unidades motrices de carga

4. Número de empresas de transporte internacional

5. Licencias internacionales 
c) Recursos humanos

6. Conductores capacitados

d) Calidad

7. Centros de capacitación

8. Número de inspecciones realizadas

9. Número de verificaciones

\section{III.2. Outputs}

a) Valor de las exportaciones

Por su parte, la selección de outputs obedece al fin último de las operaciones de comercio exterior: un mayor flujo de productos en los mercados internacionales, por lo que será importante medir el nivel de comercio internacional que se tiene particularmente a través de las exportaciones considerando el valor de la mercancía comercializada.

\section{RESULTADOS}

Los resultados del análisis realizado a través de modelo DEA y el Índice de Malmquist, se presentan en el cuadro 1 a partir de la columna tres (C3).

Las columnas C3 a la C7 muestran el grado de eficiencia de los estados analizados para los periodos $2010 \mathrm{al} 2014$, respectivamente. Las entidades que mostraron una eficiencia constante en el periodo estudiado son Aguascalientes, Baja California Sur, Campeche, Chiapas, Chihuahua, el Estado de México, Guerrero, Morelos, Nayarit, Nuevo León, Oaxaca, Quintana Roo, Tlaxcala y Zacatecas. Mientras que algunos estados no mostraron tener eficiencia para ninguno de los años parte del estudio como es el caso de Hidalgo, Jalisco, Michoacán, Puebla, Querétaro, San Luis Potosí, Sinaloa, Sonora, Tamaulipas y Veracruz. Esto se corrobora en la columna C8 donde se muestra la media geométrica de la eficiencia de todos los periodos (2010-2014) ya que en orden ascendente el valor de las cinco medias más bajas lo obtuvieron Sinaloa, Michoacán, Hidalgo, Durango, San Luis Potosí, Colima y Veracruz; siendo los valores máximos y mínimos de las entidades no eficientes 0.9949 (Coahuila) y 0.0195 (Sinaloa).

Cuatro Índices de Malmquist se obtienen al tomar como referencia primero la frontera 1 (2010) y posteriormente la frontera 2 (2012) y así sucesivamente hasta el año 2014 adoptando los métodos de análisis de Caves, et al. (1982) para el cálculo del Índice Malmquist, Fare et al. (1992). Los resultados de dichos índices se 
pueden observar en las columnas $\mathrm{C} 9$ a la $\mathrm{C} 12$, que muestran fronteras adyacentes como una frontera conjunta o frontera-bienal. La fórmula base para la frontera de dichas columnas es Eficiencia (2014-1) $=E^{2013 U 2014}\left(x^{2014}, y^{2014}\right)$ dicho de otro modo la columna C12 toma como referencia la frontera de eficiencia para el año 2014.

Los valores más importantes resultados del análisis DEA Malmquist plasmados en la tabla 1 son los valores del Índice Malmquist (C13).

El modelo utilizado se basa en el concepto de eficiencia técnica como objetivo del cambio tecnológico que en algunos sectores juega un papel clave para la mejora de la productividad. Justamente los valores obtenidos del cambio tecnológico (C13) permiten observar el grado de proceso en la tecnología empleada en el sistema de transporte carretero nacional de carga.

El Índice de Malmquist genera una frontera de posibilidades de producción para cada periodo, en este caso 2010 y 2014, lo que nos permite analizar los cambios en la productividad de cada una de las DMUS en los periodos. La medición del Índice de Malmquist representa el crecimiento de la productividad total de los factores de una unidad de toma de decisiones, en este caso del sistema de transporte de carga por entidad federativa, en donde se refleja el progreso o retroceso en la eficiencia y, por otro, el progreso o retroceso de la frontera tecnológica entre dos periodos de tiempo bajo el marco de múltiples entradas y salidas (Cooper, Seiford, \& Karou, 2007). De tal manera que un coeficiente mayor a 1 representa un cambio o progreso en la productividad total de los factores de la unidad analizada, un valor igual a 1 representa un valor constante en la productividad y finalmente un valor inferior a 1 representa un deterioro en la productividad total de los factores. 


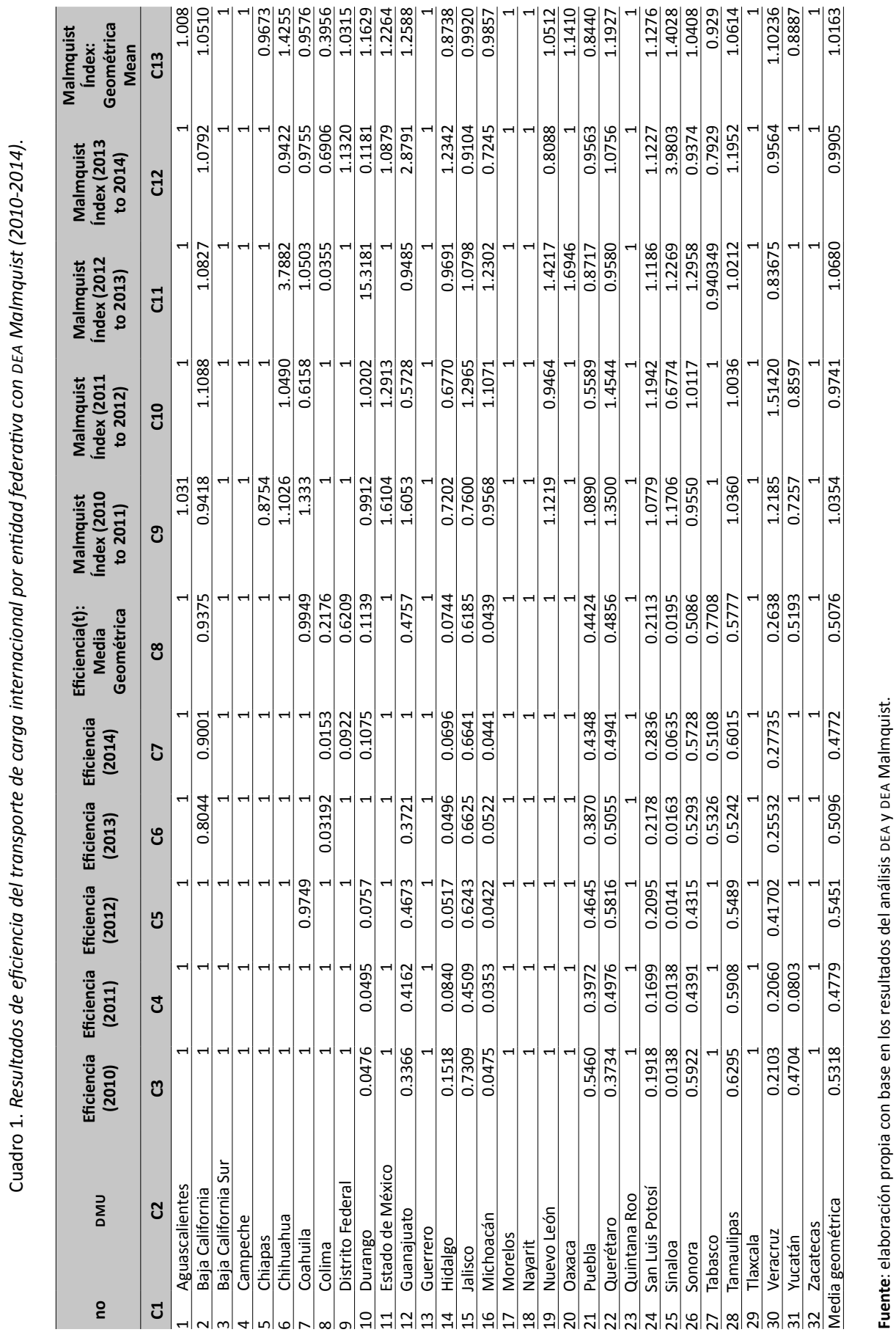


Por tanto, los resultados del Índice de Malmquist, para el presente análisis muestran que las entidades federativas con el sistema carretero de carga con un mayor cambio tecnológico son, en orden descendiente: Chihuahua, Sinaloa, Guanajuato, El Estado de México, Querétaro, Durango, Oaxaca, San Luis Potosí, Veracruz, Tamaulipas, Nuevo León, Baja California, Sonora, el Distrito Federal y Aguascalientes (véase figura 1).

Mientras que las entidades que mostraron un valor constante en la productividad fueron: Baja California Sur, Campeche, Guerrero, Morelos, Nayarit, Quintana Roo, Tlaxcala y Zacatecas. Dicho resultado no implica necesariamente que sea algo negativo siempre y cuando el avance tecnológico del transporte carretero esté en la punta de lanza y no sea necesario un incremento en la productividad. Sin embargo, si no fuese ese el caso, este indicador estaría reflejando un estancamiento.

Finalmente, las entidades que muestran un retroceso en la eficiencia respecto al sistema de transporte de carga internacional, ya sea por una desactualización o menor mantenimiento de los inputs son, de menor a mayor puntuación: Colima, Puebla, Hidalgo, Yucatán, Tabasco, Coahuila, Chiapas, Michoacán y Jalisco.

Figura 1. Índice Malmquist de transporte carretero de carga por entidad federativa.

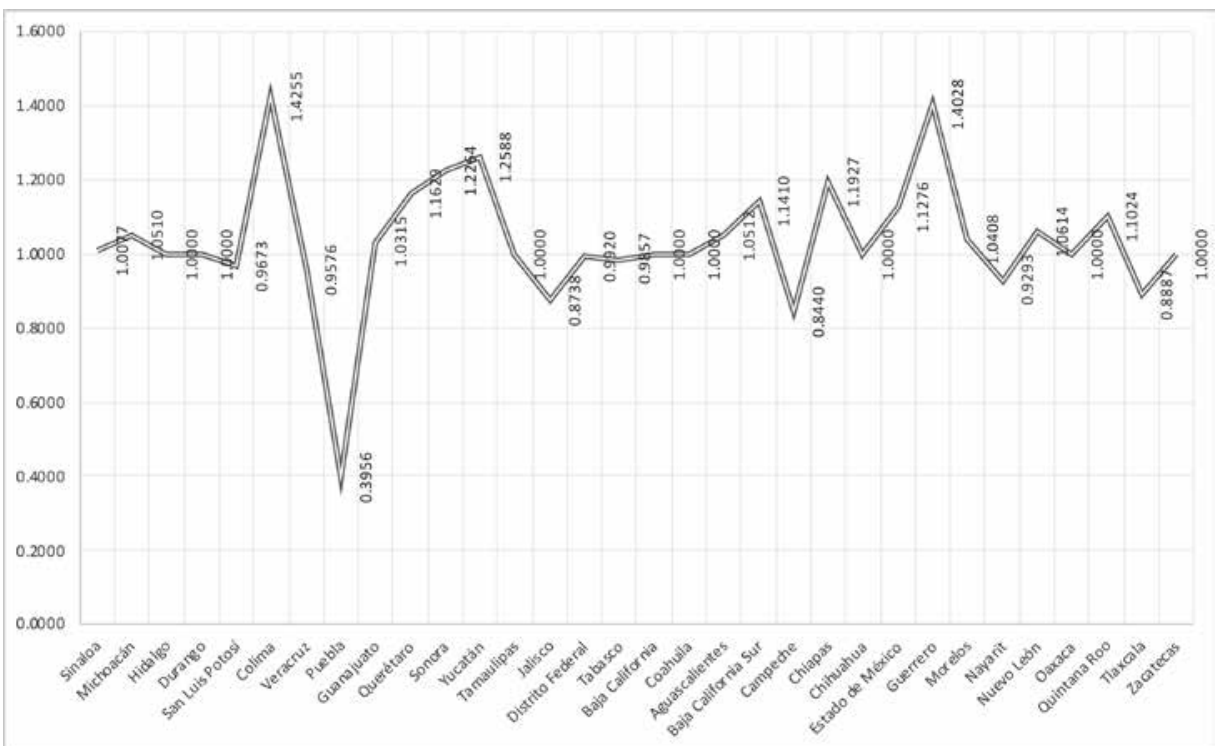

Fuente: elaboración propia con base en los resultados del análisis DEA y DEA Malmquist. 


\section{CONCLUSIONES}

Actualmente, el transporte de carga internacional es una de las variables más relevantes, implícitas en el análisis del comercio internacional; incluso puede ser de las más importantes, particularmente tratándose de exportaciones mexicanas. De ahí la relevancia en identificar qué entidades federativas han mostrado una mejora en la productividad y así obtener el componente de cambio tecnológico. Los resultados obtenidos muestran el progreso tecnológico en el periodo revisado dando cuenta de una media geométrica de 1.0163. No obstante, no todas las entidades federativas han mostrado un avance en este sentido, lo que manifiesta una disparidad importante en cuanto a variables como inversión en infraestructura logística carretera así como capacitación y tecnificación del sistema de transporte de carga mexicano.

Cabe destacar que la mejora tecnológica del eslabón de transporte, dentro del marco del comercio exterior debe ser una sinergia de inversión privada y gubernamental diseñada a partir de estrategias que conlleven a un crecimiento del sector exportador, así como una mejora significativa en la competitividad del mismo.

Adelantando algunas conclusiones puntuales, se puede afirmar que, a través del presente trabajo, se logró identificar el grado de eficiencia (ineficiencia) del sistema de transporte carretero de carga internacional a nivel de entidades federativas de México, para el periodo 2010-2014. El cuadro 1 permite identificar el grado de eficiencia o ineficiencia de cada una de las entidades federativas.

$\mathrm{Al}$ analizar las debilidades de algunos de los estados peor evaluados se encuentra los siguientes puntos a resaltar: Guerrero mostró una baja densidad vehicular de carga, denotando una baja actividad comercial en general; Campeche tuvo los valores más bajos en cuanto al número de unidades motrices de carga y número de licencias internacionales para el transporte de carga, lo que se puede traducir en poca capacidad de inversión privada para este sector. Nayarit, mostró el menor número de empresas dedicadas al transporte internacional.

Respecto a la variable calidad, los estados con más bajos resultados son Baja California y Zacatecas que no cuentan con centros de capacitación, mientras que, los estados de Nuevo León, Quintana Roo, Tabasco y Zacatecas, no realizan inspecciones y verificaciones.

Otro de los objetivos del análisis fue determinar el cambio tecnológico en cada una de los estados, tomando como referencia del cambio tecnológico el año 2010. El resultado (columnas C9 y C12) indica que Chihuahua, Sinaloa, Guanajuato, Estado de México, Querétaro, Durango, Oaxaca, San Luis Potosí, Veracruz, Tamaulipas, Nuevo León, Baja California, Sonora, CDMX y Aguascalientes son las entidades federativas con mejores resultados, en este sentido. Dichos resultados muestran que los procesos de innovación y cambio tecnológico, son fundamenta- 
les para la mejora de la productividad y que, a pesar de que no son el único factor que puede incidir en la mejora de eficiencia, son factores altamente impactantes.

Dentro de los diferentes eslabones de la cadena logística del comercio exterior, uno de los más importantes es sin lugar a dudas el medio de transporte mediante el cual se trasladarán las mercancías de un punto a otro; por lo que la mejora del transporte carretero de carga por sí misma representa una mejora significativa en la competitividad de todos los productos de exportación transportados por este medio lo que se traduce en una mejora en el posicionamiento comercial de México en la economía internacional. De ahí la relevancia de identificar qué tanto los elementos que lo componen pueden ser susceptibles de posibles mejoras en cada uno de ellos, con la finalidad de realizar mejoras estratégicas en su productividad e incidiendo, por ende, en el incremento sostenido de su eficiencia.

$\mathrm{Al}$ analizar las implicaciones de política es importante resaltar que el cambio tecnológico en vías y transporte depende, la mayoría de veces, de políticas federales, incluso de decisiones de empresas multinacionales; por ello, cabe mencionar los mecanismos o alcances que tienen las políticas estatales para mejorar los indicadores de transporte de carga. En este sentido resulta importante mencionar que las entidades federativas tienen diversos mecanismos a través de los cuales, si así lo consideran los gobiernos estatales, pudieran mejorar la eficiencia carretera; dentro de estos mecanismos se consideran los planes estatales de desarrollo, así como los programas sectoriales de infraestructura y obra pública, que son clave para hacer efectiva una determinada política pública en todas sus escalas que busque como fin último mejorar al sistema de transporte de carga nacional para incentivar el comercio internacional.

\section{REFERENCIAS BIBLIOGRÁFICAS}

Asociación Nacional de Transporte Privado (ANTP) (2018), "Estadística de robo al transporte de carga durante el 2018”, Revista Transporte e Industria (TI), núm. 101, año 19. México, pp. 2-5.

Arvis, Jean (2007), Connecting to compete-Trade Logistics in the Global EconomyThe Logistics Performance Index and its Indicators, Banco Mundial.

Bardhan, Pranab (1995), The Contributions of Endogenous Growth Theory to the Analysis of Development Problems: An Assessment, C. F. (CIDER), ed., working papers C94-038.

Beltrán-Esteve, Mercedes y Picazo-Tade, Andrés (2015), “Assessing Environmental Performance Trends in the Transport Industry: Eco-Innovation or Catching-up?", Energy Economics, vol. 51, september, pp. 570-580. 
Berg, Sigbjorn; Forsund, Finn y Jansen, Eilev (1992), Malmquist Indices of Productivity Growth During the Deregulation of Norwegian Banking, 1980-1989, Scand J. Econ. 94, S211-S228.

Caves, Douglas; Christensen, Laurits y Diewert, Walter (1982), "The Economic Theory of Index Numbers and the Measurement of Input, Output, and Productivity", Econométrica, 50 (6), pp. 1393-1414.

Cervantes, S. (junio de 2011), "México de los más inseguros para transporte de carga", en AL. $\mathrm{t} 21$.

Çipil, Fatih (2016), “Total Factor Productivity Changes of Turkey's Regional Aitr Transportation Using dea with Malmquist Index", Journal of Polytechnic, Politeknik Dergisi, 19 (1), pp. 85-99.

Cipoletta Tomassian, Georgina; Pérez Salas, Gabriel y Sánchez, Ricardo (2010), Políticas integradas de infraestructura, transporte y logística: experiencias internacionales y propuestas iniciales, Cepal.

Choi, Jeasung; Roberts, David y Lee, Eunsu (2015), "Productivity Growth in the Transportation Industries in the United States: An Application of the dea Malmquist Productivity Index", American Journal of Operations Research, 5, pp. 1-20, http://dx.doi.org/10.4236/ajor.2015.51001.

Cooper, William; Seiford, Lawrence y Tone, Kaoru (2007), Data Envelopment Analysis. A Comprehensive Text with Models, Applications, References and Dea-solver Software, 2a. ed., Springer.

(2006), Introduction to Data Envelopment Analysis and Its Uses, Springer, Nueva York.

Djankov, Simeon; Freund, Caroline; Pham, Cong (Abril 2007), Trading on Time, World Bank Policy, papel de trabajo 3909.

Dussel Peters, Enrique (2008), Los costos de transporte en las exportaciones mexicanas, Universidad Nacional Autónoma de México, Banco Interamericano de Desarrollo (BID) y Centro de Estudios Latinoamericanos David Rockefeller, Harvard University.

Estadísticas de Transporte de América del Norte. (2011), obtenido de http://nats. sct.gob.mx.

Färe, Rolf y Lovell, Knox (1978), "Measuring the Technical Efficiency of Production", Journal of Economic Theory, 19, pp. 150-162.

Fare, Rolf, et al. (1992), "Productivity Change in Swedish Pharmacies 1980-1989: A Nonparametric: Approach Malmquist", Springer Journal of Productivity Analysis, núm. 3, pp. 85-101. DOI: 10 1007/BF00158770.

Farrell, M. J. (1957), "The Measurement of Productive Efficiency", Journal of the Royal Statistical Society, vol. 120, part III. 
Fisher, Ronald Aylmer (1922), On the Mathematical Foundations of Theoretical Statistics, Philosophical Transactions of the Royal Society of London, Series A, Containing Papers of a Mathematical or Physical Character, vol. 222, pp. 309-368.

Foro Económico Internacional (2017), "Índice de Competitividad Global del Foro Económico Mundial 2017-2018”, Ranking Mundial de Infraestructura.

Gonzalez, Julio; Guasch, José Luis y Serebrisky, Tomas (2007), Latin America: Addressing High Logistics Costs and Poor Infrastructure for Merchandise Transportation and Trade Facilitation, World Bank.

Grifell, Emili y Lovell, Knox (1993a), Deregulation and Productivity Decline: The Case of Spanish Saving Banks, working paper, 93-02 (june), Department of Economics, University of North Carolina.

(1993b), A New Decomposition of the Malquimist Productivity Index, working paper, 93-04 (october), Department of Economics, University of North Carolina.

(1996), "Deregulation and Productivity Decline: The Case of Spanish Saving Banks", European Economic Review, 40, pp. 1281-1303.

IEA/OECD (2009), Transport, Energy and CO2. Moving Toward Sustainability, IEA/ OECD, Paris.

Kim, Nam y Van Wee, Bert (2009), "Assessment of $\mathrm{CO}_{2}$ Emissions for Truck-only and Rail-Based Intermodal Freight Systems in Europe", Transportation Planning and Technology, 32 (4), pp. 313-333.

Mahammady, Al-Rousan (2006), "A Data Envelopment Analysis Approach Based on Total Cost of Ownership for Supplier Selection", Journal of Enterprise Information Management, vol. 19, núm. 6, pp. 662-678.

Malmquist, Sten (1953), "Index Numbers and Indifference Surfaces", Trabajos de Estadística, 4, pp. 209-242.

Martínez, Rivas Marelia (2008), Hacia una política nacional de logística, Taller de presentación de resultados del diseño conceptual de un esquema de sistemas de plataformas logísticas en Colombia. Hacia una política nacional de logística, Bogotá.

Medina Ramírez, Salvador (2011), Apertura fronteriza al transporte de carga mexicano: ¿Fin del problema?, Comercio Exterior.

Mendoza Cota , Jorge Eduardo y Díaz, E. (2003), “Obstáculos al comercio en el TLCAN: El caso de transporte de carga”, Comercio Exterior, vol 53., núm. 12., pp. 1112-1120.

Moorstenn, R. H. (1961), "On Measuring Productive Potential and Relative Efficiency", Quarterly Journal of Economics, 75, pp. 451-467.

Nishimizu, Mieko y Page, John (1982), “Total Factor Productivity Growth, Technological Progress and Technical Efficiency Change: Dimension of Productivity Change in Yugoslavia, 1965-78”, The Economic Journal, 92, pp. 920-936. 
OMC (2013b), Organización Mundial de Comercio, disponible en http://www. wto. org/indexsp.htm.

Programa Sectorial de Comunicaciones y Transportes 2013-2018 (2013), "Plan Nacional de Desarrollo del Gobierno de la República", Gobierno de la República, Ciudad de México.

Sarmento, Joaquim; Renneboog, Luc y Verga, Pedro (2017), "Measuring Highway Efficiency by a DEA Approach and the Malmquist Index", EJTIR, Issue 17 (4), pp. 530-551.

Secretaría de Comunicaciones y Transportes (SCT) (2013), Plan Nacional de Desarrollo 2013-2018. Programa Sectorial de Comunicaciones y Transportes 20132018, México, 130 pp.

(2014), Secretaría de Comunicaciones y Transportes, obtenido de título de concesión que en materia Ferroviaria, ha otorgado el Gobierno Federal, por conducto de la Secretaría de Comunicaciones y Transportes, http://gaceta.diputados.gob.mx/Gaceta/62/2014/feb/ConseFerr-20140218.pdf.

(2015), «Secretaría de Comunicaciones y Transportes», obtenido de: http://www. sct.gob.mx/fileadmin/DireccionesGrales/DGAF/Documentos/03_carga.pdf. (2016), «Principales Estadísticas del Sector Comunicaciones y Transportes. Ciudad de México».

Secretaría de Medio Ambiente y Recursos Naturales (2014), «Teoría y conceptos generales para elaborar inventarios verificables», Subsecretaría de Fomento y Normatividad Ambiental. Coordinación de Asesores, México.

Schøyen, Halvor y Odeck, James (2015), "Comparing the Productivity of Norwegian and Some Nordic and uk Int. J. Shipping and Transport Logistics", Inderscience Publishers Ltd. This is an Open Access Article Distributed Under the cc by-ncnd, license, vol. X, núm. Y, pp. 1-23.

Törnqvist, L. (1936), The Bank of Finland's Consumption Price Index, Bank of Finland Monthly Bulletin, núm. LO, pp. 1-8

Wanke, Peter; Chen, Zhongfei; Moreira, Jorge Junio y Barros, Carlos (2018), "Malmquist Productivity Indexes in Chinese Ports: a Fuzzy gmss dea Approach", International Journal of Shipping and Transport Logistics (IJSTL), vol. 10, núm. 2.

Wilmsmeier, Gordon, \& Sanchez, Ricardo (2009), Los desafíos del sistema de transporte en los países sin litoral de América del Sur, Cepal.

Zhang, Ning; Zhou, Peng; Kung, Chin-Chun (2015), “Total-factor Carbon Emission Performance of the Chinese Transportation Industry: A Bootstrapped Non-radial Malmquist Index Analysis", Renewable and Sustainable Energy Reviews, vol. 41, january, pp. 584-593. 